within the health sector and routes into key decision-making areas within government are critical issues that need to be addressed.

NSW Health has committed considerable resources to pursuing the potential of HIA. Harris-Roxas and Harris in their articles outline the work of the NSW HIA project coordinated by the Centre for Health Equity Training, Research and Evaluation. This project has supported developmental sites to use a 'learning by doing' approach to gain experience in applying HIA. Articles in this issue (Thackway et al and O'Hara et al) reflect upon the outcomes and better place us to make strategic decisions on how to maximise the potential gains from employing HIA in a NSW context.
HIA can be a useful tool in minimising the negative impacts and enhancing the positive impacts of policies and projects. HIA can be undertaken at a number of levels-as a deskbased audit, rapid assessment and comprehensive HIA. It is also only one of many assessment processes including Aboriginal health impact assessment (Wheeler), and social and environmental impact assessments. As we move to working more closely with other government agencies and the private sector there will be pressure to find ways of developing integrated impact assessment processes. The relative merits of creating a separate HIA process, including looking at the different contexts in which it may operate, need to be reviewed now that we have had some experience with the procedures, methods and tools that HIA uses. 뭏

\title{
AN INTRODUCTION TO HEALTH IMPACT ASSESSMENT
}

\section{Sarah Simpson*}

Centre for Health Equity Training, Research

and Evaluation

University of New South Wales

Health impact assessment (HIA) is a structured and stepwise process for identifying the potential beneficial and harmful health impacts of a policy, program, development or project proposal before its implementation, with the purpose of using this information to improve the proposal. A widely used and accepted definition of HIA is:

A combination of procedures, methods and tools by which a policy, program or project may be assessed for its potential, and often unanticipated, effects on the health of the population and the distribution of these impacts within the population. ${ }^{1,2}$

HIA has its roots in other forms of assessment such as environmental impact assessment and health risk assessment. $^{3}$ These roots are reflected in the steps of HIA, which are generally understood to include:

1. Screening - is an HIA required?

2. Scoping - setting the boundaries of the HIA.

3. Identification of impacts - collecting information from agreed sources about potential health impacts.

4. Assessment of impacts - assessing the range of identified health impacts from the different sources.

5. Development of recommendations - to improve the proposal by amelioration of potentially negative health impacts and/or enhancement of potentially positive health impacts.

* Sarah Simpson is currently employed by the World Health Organization in Geneva as Coordinator, Knowledge Networks, Commission on the Social Determinants of Health
6. Evaluation and monitoring -includes (a) evaluation of the HIA process, (b) setting up a structure to monitor the impact of the HIA, including uptake of recommendations, and (c) assessing the health impact of the proposal following implementation. ${ }^{4}$

The focus of other forms of impact assessment such as environmental impact assessment is usually on major infrastructure developments such as a new road or extending an airport, and information is collected with a view to protecting the environment and/or human health. These assessments usually draw on a more circumscribed evidence base (for example systematic reviews) and use a 'tight' definition of health ${ }^{5}$ to guide regulatory intervention that might be required to protect the environment or health.

The articles in this issue of the NSW Public Health Bulletin emphasise the broad range of contexts for HIA as an aid to decision-making, for instance for a new policy (for example family tax benefit) or a new physical development (for example construction of a port facility). Where HIA is used in this way, potential health impacts are usually identified using a wide range of sources (for example focus groups and the literature), the focus is on protecting and promoting health and a 'broad' definition of health is applied. ${ }^{6} \mathrm{~A}$ wide body of literature has been published on this application of HIA (see Mahony and Durham ${ }^{7}$ for an overview).

Where HIA is used as part of the policy or program development process, it is best undertaken before the proposal is finalised (see Harris, 'Contemporary debates in health impact assessment: What? Why? When?', in this issue) so that decision makers can apply the findings before implementation. The results of HIA can be used to improve proposals developed within and outside the health sector, for example the potential impacts of changes to how health 
services are delivered in a region or the potential impacts of a change to gambling laws.

Identification of the unintended potential impacts of a proposal is a particularly important aspect of HIA because of the assumption that all proposals are well intentioned and there will be few if any negative impacts from their implementation. However, this is not always the case. For example a campaign to promote healthy lifestyles, where the intent is to improve and promote people's health, may also unintentionally stigmatise those groups in the population who are unable to act on the information (for a range of reasons not within their control).

Values such as transparency, equity and community participation are generally accepted as integral to the HIA process. ${ }^{8}$ Practitioners, however, have found that the incorporation of these values can be challenging in practice (see Aldrich et al, 'Building an equity focus in health impact assessment' in this issue).

Health impact assessment is not a mandated part of the policy or program development process in any state or territory in Australia. ${ }^{6}$ However, there is increasing interest in the use of HIA as a method for improving practice and there are an increasing number of Australasian examples of its applications. As described by Harris-Roxas and Simpson in 'The NSW Health Impact Assessment Project' in this issue, in NSW there is a strong commitment to building the capacity to use HIA to improve policies, programs and projects that are developed by health and, more recently, proposals by other sectors.

\section{REFERENCES}

1. European Centre for Health Policy. Health impact assessment: Main concepts and suggested approaches. Gothenburg consensus paper. Brussels, ECHP \& World Health Organization, Regional Office for Europe: Copenhagen, 1999.

2. Mahoney M, Morgan R. Health impact assessment in Australia and New Zealand: an exploration of methodological concerns. International Journal of Health Promotion and Education 2001; VIII(1): 8-11.

3. Mahoney M. Current thinking and issues in the development of health impact assessment in Australia. NSW Public Health Bulletin, 2002; 13(7): 167-9.

4. Simpson S, Harris E, Harris-Roxas B. Health impact assessment: An introduction to the what, why and how. Health Promot J Austr, 2004; 15(2): 150-5.

5. Kemm J. Can health impact assessment fulfil the expectations it raises? Public Health 2000; 114(6): 431-3.

6. Harris E, Simpson S. NSW Health Impact Assessment Project. Phase 1 Report. Sydney: CHETRE, School of Public Health and Community Medicine, University of NSW, 2003.

7. Mahoney M, Durham G. Health impact assessment: a tool for policy development in Australia. Victoria: Faculty of Health and Behavioural Sciences, Deakin University, 2002.

8. Douglas MJ, Conway L, Gorman D, Gavin S, Hanlon P. Developing principles for health impact assessment. J Public Health, 2001; 23(2): 148-54. 용

\section{CONTEMPORARY DEBATES IN HEALTH IMPACT ASSESSMENT: WHAT? WHY? WHEN?}

\author{
Elizabeth Harris \\ Centre for Health Equity Training, Research \\ and Evaluation \\ University of New South Wales
}

Public health practitioners and policy makers in Australia have a long history of advocating for health impact assessment (HIA) to be undertaken on major public and private projects and policies. Over the past decade a number of internationally recognised guidelines and discussion papers have been developed in Australia that have tried to strengthen HIA in environmental impact assessment processes. ${ }^{1,2}$ Internationally, advocates for the development of healthy public policy have promoted HIA as a necessary step in policy development. ${ }^{3,45,6}$ Those with an interest in reducing health inequalities have supported this and called for a closer examination of the distributional impacts on groups within the population, especially those groups most marginalized or disadvantaged. ${ }^{7,8,9,10}$

As outlined elsewhere in this issue of the NSW Public Health Bulletin, there have now been significant investments by most Australian jurisdictions in developing HIA capacity within the health system. As these initiatives unfold there are a number of issues that need to be understood and debated within the public health community. These involve the what, why and when of HIA. This article presents those issues and Staff, in the article that follows, provides a response to each of these issues.

\section{WHAT IS HIA?}

HIA is a structured process for assessing the impacts of a proposed policy, program or project on health at the point when it has been sufficiently developed to allow likely impacts to be determined. ${ }^{11}$ HIA is therefore only one of several strategies that need to be put in place to ensure consideration of the impacts on health of what society does generally. Needs assessment, planning, monitoring and evaluation also play important roles in promoting and protecting health in the planning and development process. $^{12}$

Issue: As well as promoting HIA as an effective tool for assessing impacts on health, we also need to identify ways of building considerations of health impact earlier in the policy or project development process. 